argon ratios for a tuff in the Lower Unit. This dated tuff, however, is overlain by an unconformity of unknown duration and this means that the age of the Upper Unit is not known with certainty. The suggestion that the dated tuff at Koobi Fora is contemporary with a similar tuff at Ileret, approximately 30 miles away, must also be viewed with caution.

The complex and fascinating picture of hominid history which is emerging at East Rudolf poses a variety of questions. Although none of these questions is really new, perhaps enough material is now becoming available so that answers may be proposed with more assurance than before. - From our Palaeoanthropology Correspondent.

\section{HEPATITIS}

\section{Model in Monkeys}

from our Medical Virology Correspondent

THE variety of animals which have been used to find a suitable experimental model for viral hepatitis include hamsters, horses, African gerboas, Mongolian gerbils, the egg of the domestic fowl, canaries, pigeons and non-human primates (J. W. Mosley, Arch. Ges. Virusforsch., 22, 252; 1967). F. Deinhardt and his associates, and others, have demonstrated that species of marmosets, Saguinus nigricollis, $S$. fuscicollis and $S$. mystax, can serve as animal models for human type A (infectious or epidemic) hepatitis (see Nature New Biology, 229, 130; 1971), but in the absence of serological or other markers for the elusive virus of infectious hepatitis, this particular monkey model, although undoubtedly urseful, cannot be used generally and the test system is still inadequate.

The discovery of the relationship between Australia antigen and type B (serum) hepatitis has now provided the necessary marker for the study of this form of viral hepatitis (Bull. Wld Hlth Org., 42, 957; 1970) and advances in this field have recently been rapid. William $T$. London and his colleagues from the National Institutes of Health, Bethesda (J. Infect. Dis., 125, 382; 1972), now report the successful serial transmission in rhesus monkeys (Macaca mulatta) of an agent associated with hepatitis B antigen (previously referred to as Australia antigen). The infection in these monkeys was inapparent and it was not associated with biochemical evidence of liver disease nor with obvious histological changes in the liver. Hepatitis $B$ antigen was demonstrated transiently and antibody responses were recorded in the animals inoculated with serum from a human antigen carrier with chronic hepatitis at the primary passage. The antibody responses in infected rhesus monkeys were similar in pattern to those observed in man following either natural or artificial infection with hepatitis B virus. Successful transmission was achieved through five serial passages in rhesus monkeys. Vervets (Cercopithecus aethiops), on the other hand, appeared to be refractory to this infection.

The finding of hepatitis B antigen and antibody in several nonhuman primates has been reported previously and the immunization with this antigen of chimpanzees (E. A. Lichter, Nature, 224, 810; 1969) and other species had also been recorded; but William $T$. London and his colleagues rightly believe that their observations constitute the first unequivocal demonstration of serial transmission of type B hepatitis agent in a readily available laboratory animal.

Current progress with hepatitis is therefore gratifying and the availability of suitable animal models for human hepatitis should permit the study of some of the biological characteristics and the physical and biochemical properties of these infective agents, without recourse to experimentation in human volunteers. It is perhaps also timely to recall that all nonhuman primates (as well as other animals) have an indigenous viral flora of their own and monkeys and apes must not and cannot be considered simply as "testtubes". Kalter and Heberling (Bact.
Rev., 35, 310; 1971) have recently reviewed this problem in a comprehensive report on the comparative virology of primates.

There is a striking resemblance between events in hepatitis and the historical developments which culminated in the conquest of poliomyelitis. At the beginning of the century, Landsteiner and Popper (Z. Immunitätsforsch. Exp. Ther., 2, 377; 1909) described the susceptibility to poliomyelitis virus of rhesus monkey (Macaca mulatta) and baboon (Papio hamadryas), and later other monkey species similarly were found to be susceptible to the virus. The discovery by Enders and his colleagues (Science, 109, 85; 1949; and Amer. J. Med. Sci., 220, 316; 1950 ) that tissue culture cells of primates including monkey kidney were susceptible to infection with poliovirus precipitated the large scale use of nonhuman primates in the laboratory. These observations provided the technology which led to the isolation of numerous viruses from man and animals and, ultimately, effective methods of prevention of infection were introduced.

Recent reports also offer hope for the development of tissue culture systems, which may also be suitable for the propagation in vitro of hepatitis B virus (A. B. Jenson et al., Exper. Mol. Path., 13, 217; 1970; D. H. Carver and D. S. Y. Seto, Science, 172, 1265; 1971; and A. J. Zuckerman et al., Nature, 236, 78; 1972). The prospects for active immunization of man against type B hepatitis thus seem brighter.

\title{
Two Kinds of Pulsarquake?
}

THE sudden changes in period which occur in the pulsars with shorter periods and are known as spinups or glitches seem to fall into two categories, according to D. Pines, M. Ruderman and J. Shaham. Writing in Nature Physical Science (next Monday, June 5) Pines et al. point to differences in the behaviour of the Vela and Crab pulsars at the time of glitches.

Both these pulsars seem to suffer discontinuous changes in period which can be explained in terms of starquakessudden changes in a relatively rigid rapidly spinning structure-but whereas the evidence favours a model for the Crab pulsar in which a solid crust "floats" on a core of neutron superfluid, in the case of the Vela pulsar it is more likely that the glitches arise from deformations of a solid core in a spinning neutron star.

This suggestion arises from the relatively large magnitude of the Vela spinups and their frequent occurrence, both of which require a reservoir of energy greater than that in the $C r a b$ pulsar.
Detailed changes in the periods of the two pulsars immediately following glitches also suggest that rather different mechanisms are operating in them.

What is the significance of this for pulsar models? Perhaps the principal conclusion to be drawn is that the Vela neutron star must be considerably more massive than the one in the Crab. Superfluid neutron matter solidifies only when its density exceeds $1.5 \times 10^{15} \mathrm{~g}$ $\mathrm{cm}^{-3}$, which occurs in neutron stars of mass greater than $0.6 M_{\odot}$. So the Crab pulsar must have a mass less than this critical value, and the Vela pulsar must have a mass above $0.6 M_{\odot}$. More specifically, the observed changes in the Vela spin rate, together with the model put forward by Pines et al., suggest a mass in the range 0.6 to $0.7 M_{\odot}$. And the possibility of "crustquakes" in the Vela pulsar is not ruled out, although these would probably occur independently of "corequakes" and only at intervals of several hundred years, making it unlikely that one will be observed in the near future. 